\title{
Realtime Web Apps
}

\section{With HTML5 WebSocket, PHP, and jQuery}

Jason Lengstorf

Phil Leggetter 


\title{
Realtime Web Apps: With HTML5 WebSocket, PHP, and jQuery
}

\author{
Copyright ( 2013 by Jason Lengstorf, Phil Leggetter
}

This work is subject to copyright. All rights are reserved by the Publisher, whether the whole or part of the material is concerned, specifically the rights of translation, reprinting, reuse of illustrations, recitation, broadcasting, reproduction on microfilms or in any other physical way, and transmission or information storage and retrieval, electronic adaptation, computer software, or by similar or dissimilar methodology now known or hereafter developed. Exempted from this legal reservation are brief excerpts in connection with reviews or scholarly analysis or material supplied specifically for the purpose of being entered and executed on a computer system, for exclusive use by the purchaser of the work. Duplication of this publication or parts thereof is permitted only under the provisions of the Copyright Law of the Publisher's location, in its current version, and permission for use must always be obtained from Springer. Permissions for use may be obtained through RightsLink at the Copyright Clearance Center. Violations are liable to prosecution under the respective Copyright Law.

ISBN 978-1-4302-4620-6

ISBN 978-1-4302-4621-3 (eBook)

Trademarked names, logos, and images may appear in this book. Rather than use a trademark symbol with every occurrence of a trademarked name, logo, or image we use the names, logos, and images only in an editorial fashion and to the benefit of the trademark owner, with no intention of infringement of the trademark.

The use in this publication of trade names, trademarks, service marks, and similar terms, even if they are not identified as such, is not to be taken as an expression of opinion as to whether or not they are subject to proprietary rights.

While the advice and information in this book are believed to be true and accurate at the date of publication, neither the authors nor the editors nor the publisher can accept any legal responsibility for any errors or omissions that may be made. The publisher makes no warranty, express or implied, with respect to the material contained herein.

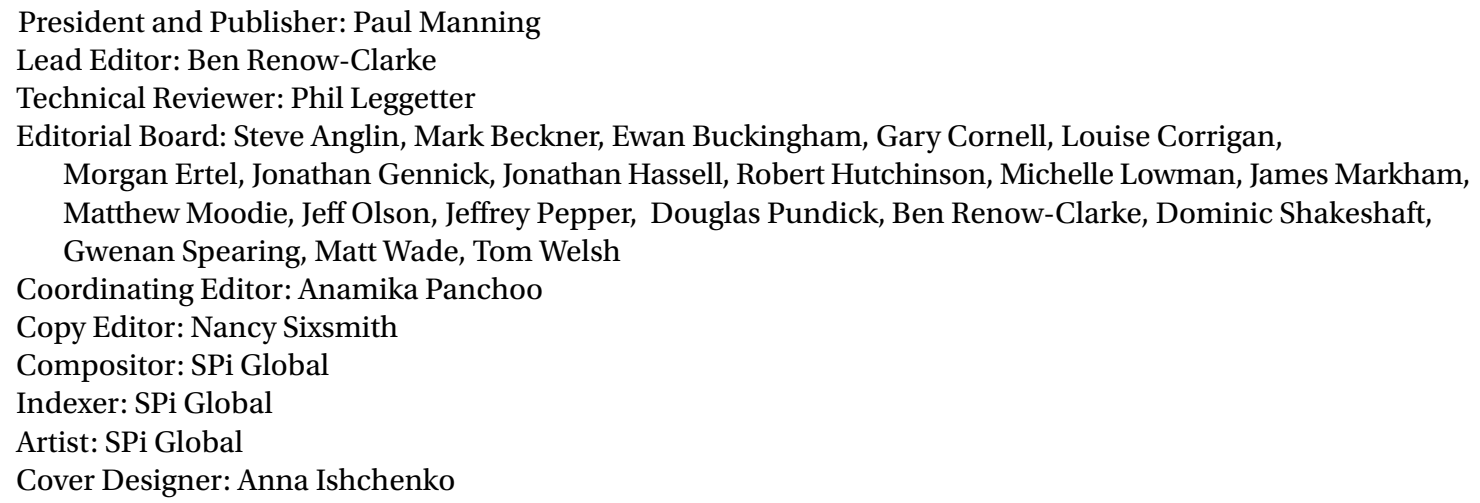

Distributed to the book trade worldwide by Springer Science+Business Media New York, 233 Spring Street, 6th Floor, New York, NY 10013. Phone 1-800-SPRINGER, fax (201) 348-4505, e-mail orders-ny@springer-sbm.com, or visit WWW. springeronline.com. Apress Media, LLC is a California LLC and the sole member (owner) is Springer Science + Business Media Finance Inc (SSBM Finance Inc). SSBM Finance Inc is a Delaware corporation.

For information on translations, please e-mail rights@apress.com, or visit www.apress .com.

Apress and friends of ED books may be purchased in bulk for academic, corporate, or promotional use. eBook versions and licenses are also available for most titles. For more information, reference our Special Bulk Sales-eBook Licensing web page at www. apress. com/bulk-sales.

Any source code or other supplementary materials referenced by the author in this text is available to readers at www. apress . com. For detailed information about how to locate your book's source code, go to www.apress.com/source-code/. 
For Tim the cat.

-Jason Lengstorf 


\section{Contents at a Glance}

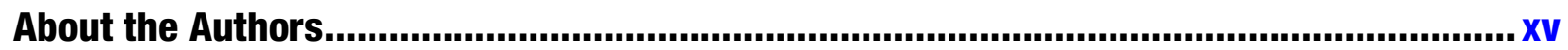

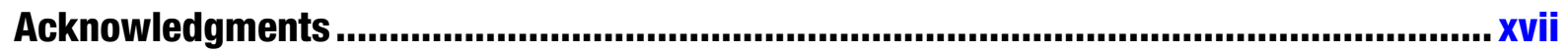

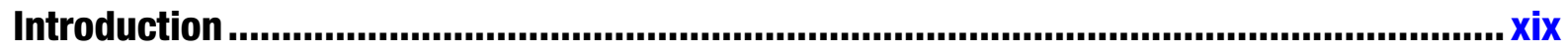

Part I: Getting Familiar with the Required Technologies .............................. 1

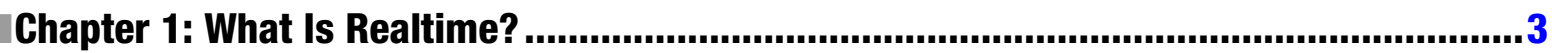

Chapter 2: The Tools ..........................................................................................15

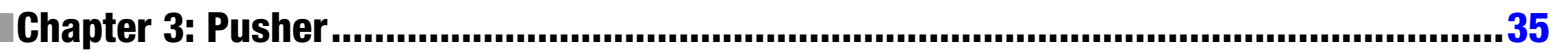

Part II: Planning the App ................................................................... 55

Chapter 4: Choosing Web Apps Over Native Apps ........................................................57

Chapter 5: Determining the App's Functionality and Structure ........................................65

Part IIl: Building the Basics............................................................. 87

Chapter 6: Designing the App .......................................................................................89

Chapter 7: Creating HTML and CSS Markup ................................................................109

Chapter 8: Building the Back End: Part 1 ................................................................155

Chapter 9: Building the Back-End: Part 2 ............................................................197

Chapter 10: Implementing Realtime Events and jQuery Effects ..................................241

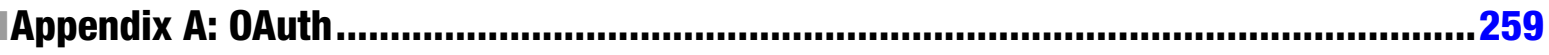

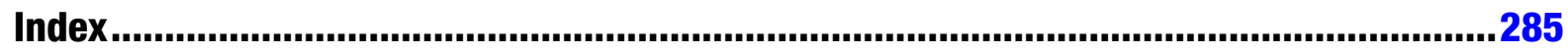




\section{Contents}

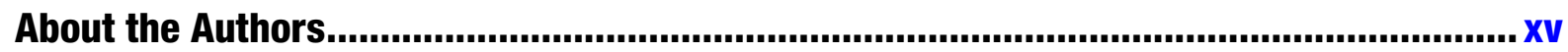

Acknowledgments ................................................................................................. xvii

Introduction ...................................................................................................................... xix

\section{Part I: Getting Familiar with the Required Technologies .......................... 1}

Chapter 1: What Is Realtime? .......................................................................................3

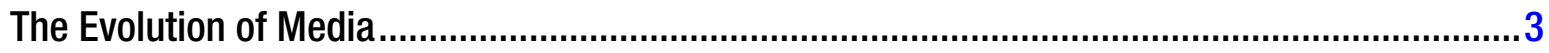

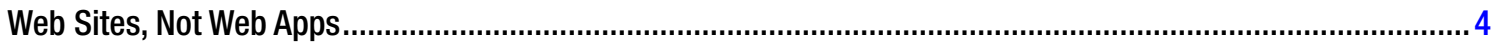

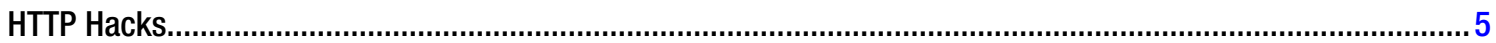

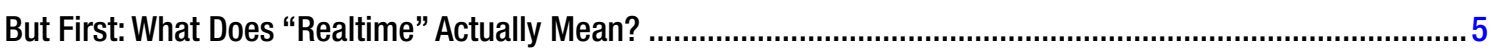

AJAX

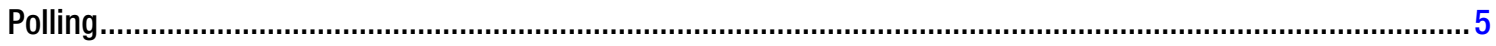

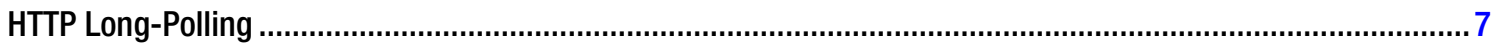

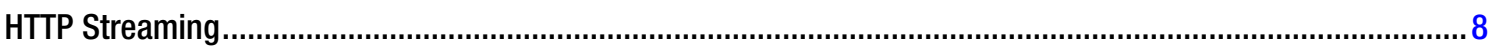

Additional Problems with HTTP-based Solutions in Web Browsers................................................................ 8

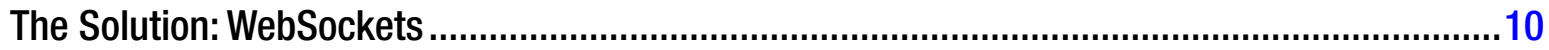

The WebSocket Protocol .................................................................................................................11

Why Bother Learning about Realtime Web Technologies? .......................................................12

Using Realtime Web Technologies in Your Apps Now ............................................................13

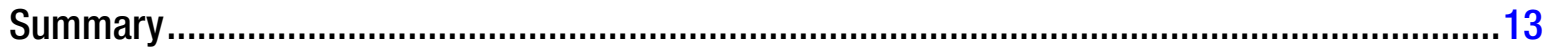

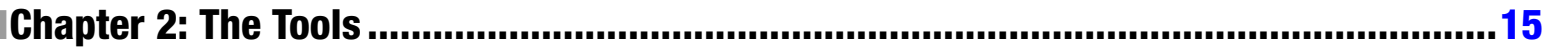

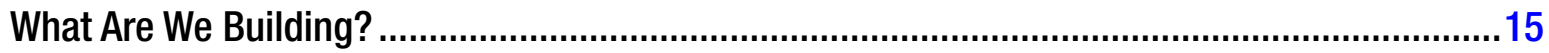

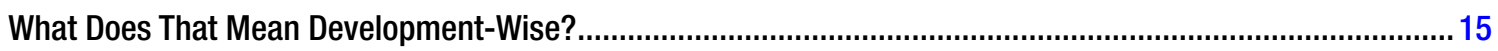

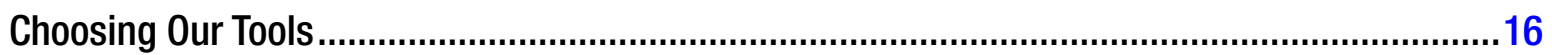




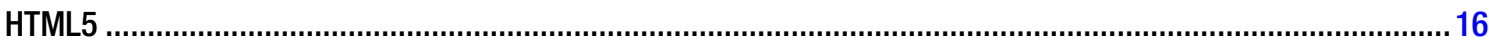

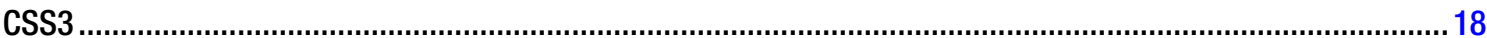

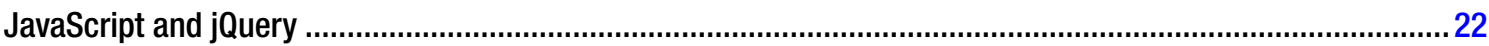

PHP

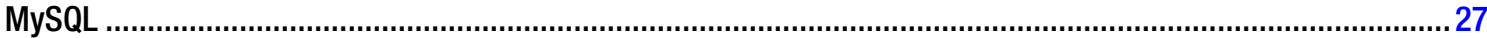

HTML5 WebSocket Technology and Pusher ............................................................................................... 30

OAuth

Summary

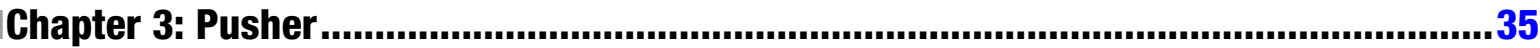

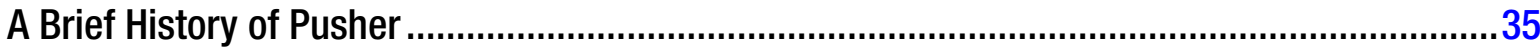

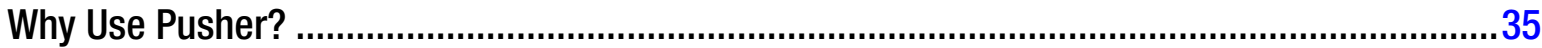

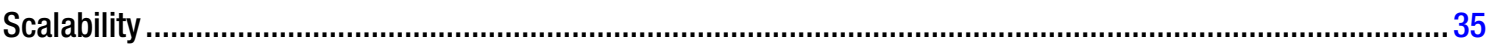

WebSocket, Fallback Support, and Auto-Reconnect................................................................................... 36

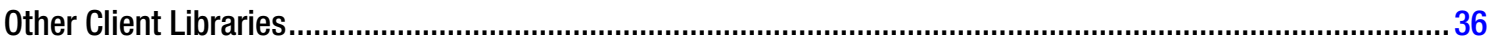

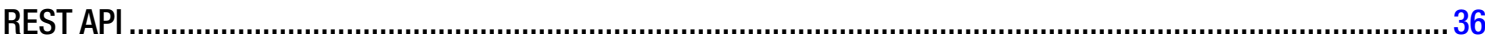

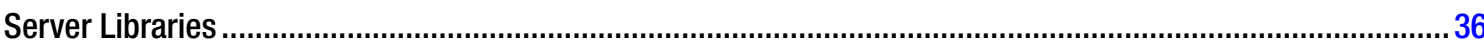

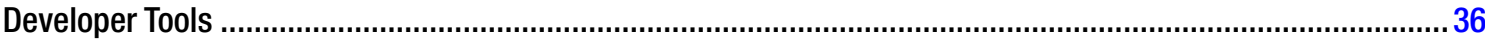

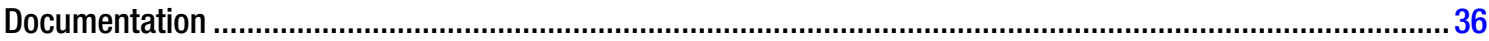

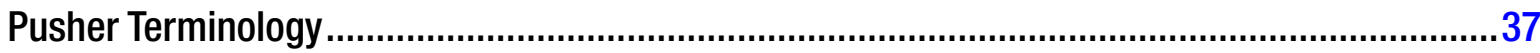

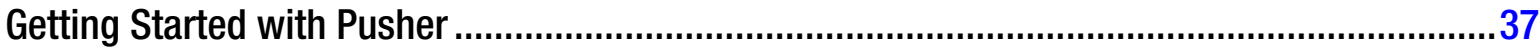

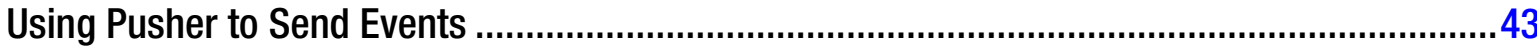

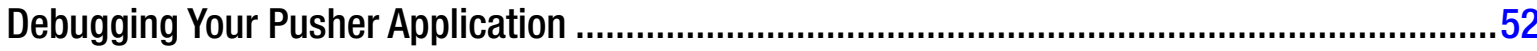

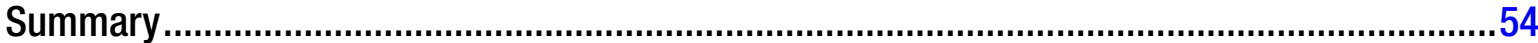

Part II: Planning the App .................................................................... 55

Chapter 4: Choosing Web Apps Over Native Apps ........................................................57

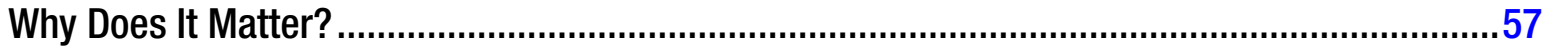

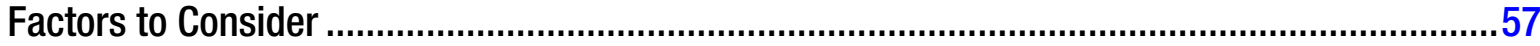

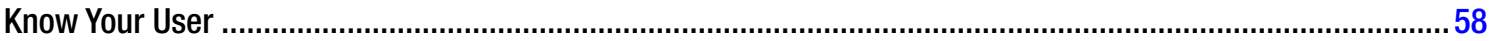

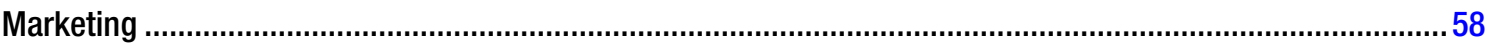

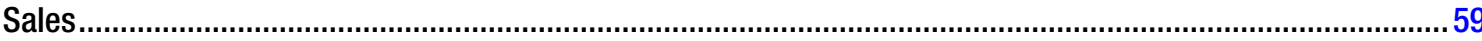


Distribution 60

Look, Feel, and Performance .60

Development. 61

Choosing Based on Requirements 63

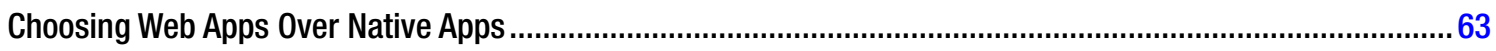

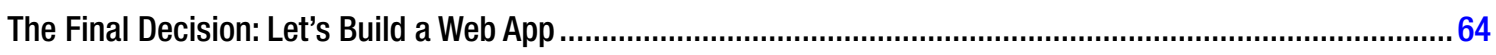

Summary.

Chapter 5: Determining the App's Functionality and Structure.................................65

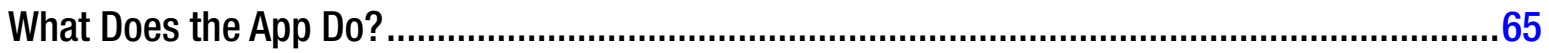

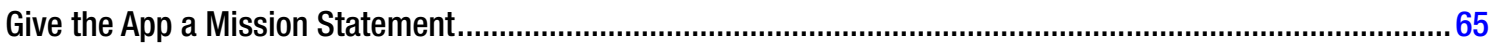

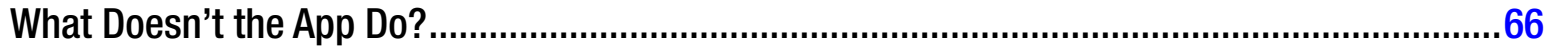

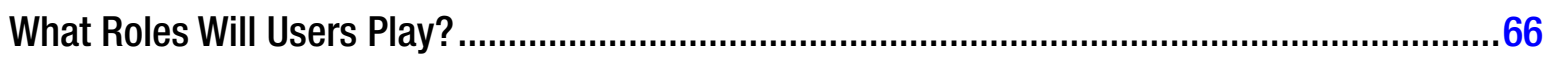

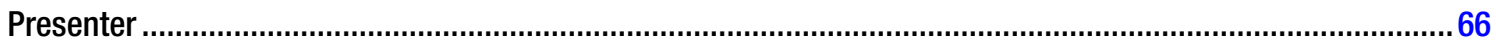

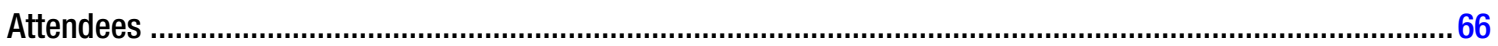

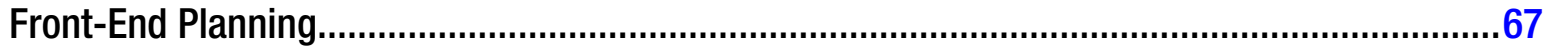

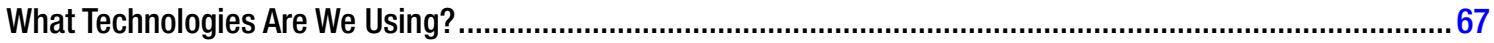

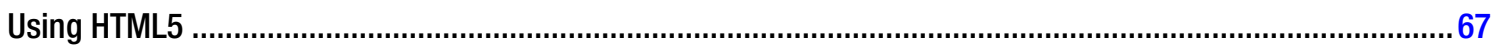

CSS3, Media Queries, and How That Affects the Design and HTML ..........................................................69

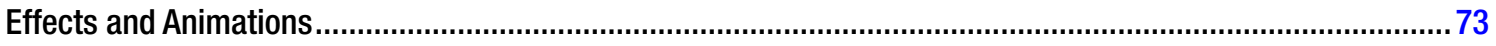

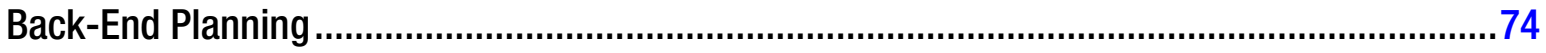

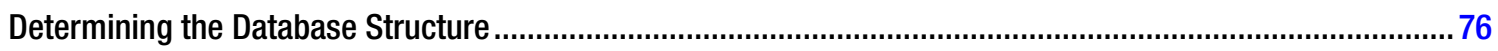

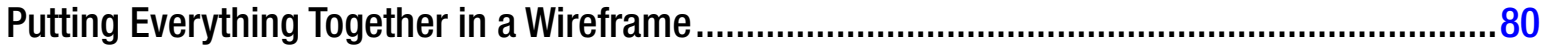

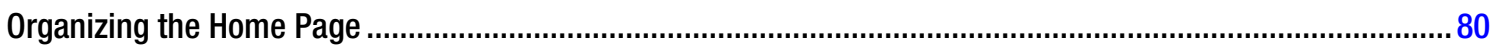

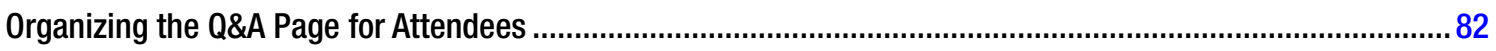

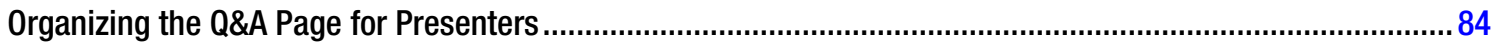

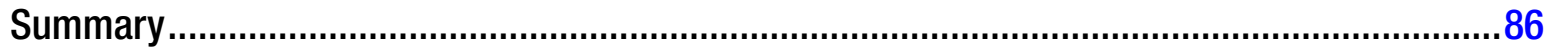

Part IIl: Building the Basics........................................................................... 87

Chapter 6: Designing the App .............................................................................89

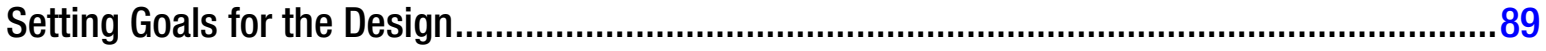

Defining the Color Palette...........................................................................................90 


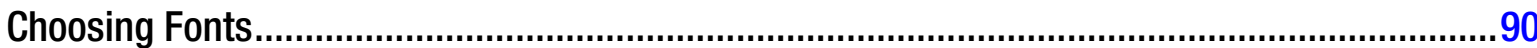

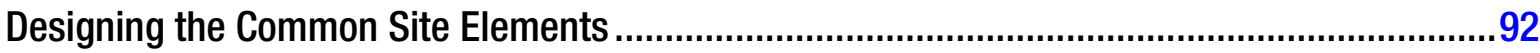

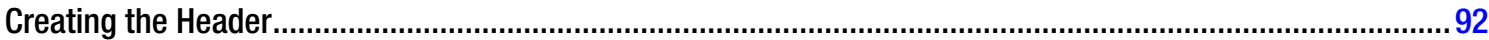

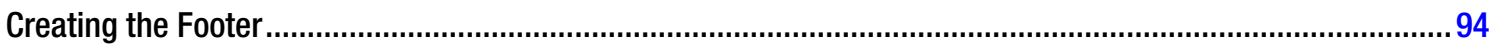

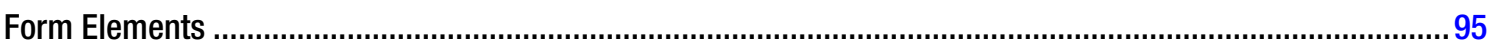

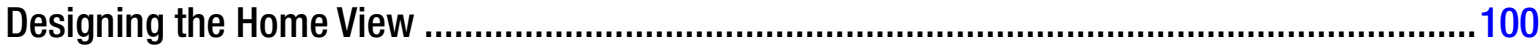

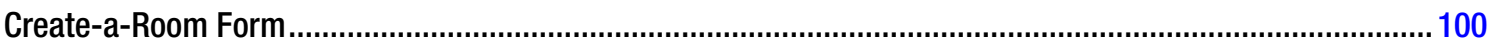

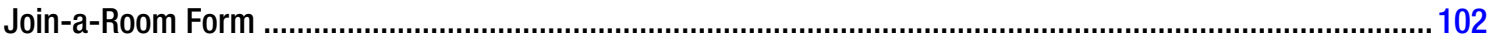

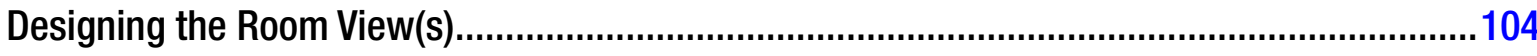

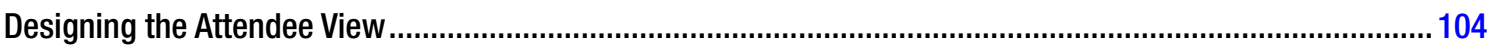

Designing the Closed Room View .................................................................................................... 106

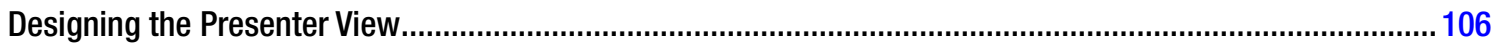

Smaller Screen Layouts (and Why You're Not Designing Them Here) ....................................108

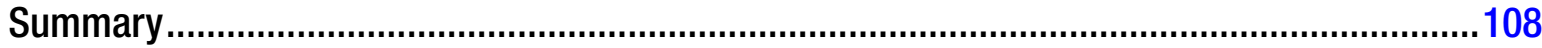

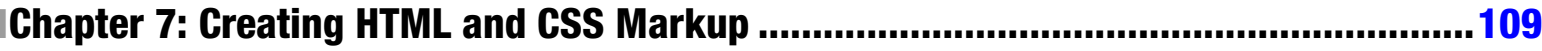

Starting with the Basics: Setting Up an HTML5 Document ...................................................109

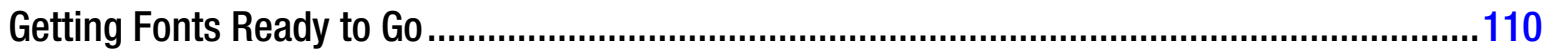

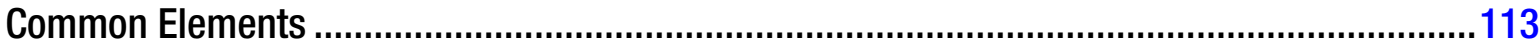

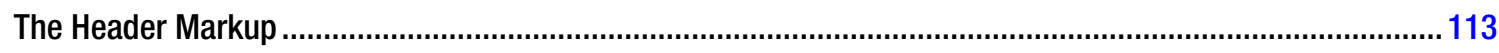

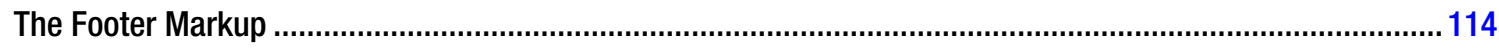

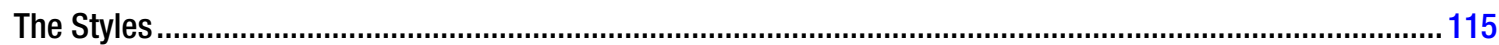

Making the Header and Footer Responsive.......................................................................................119

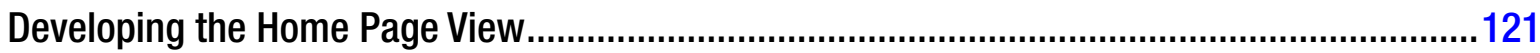

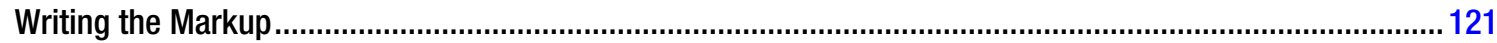

Adding Media Queries ......................................................................................................... 127

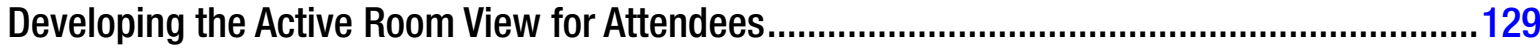

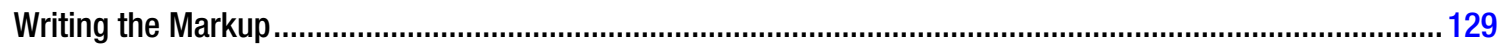

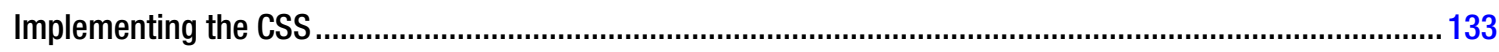

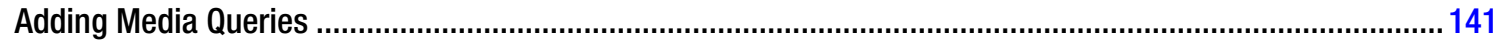


Developing the Closed Room View for Attendees..............................................................144

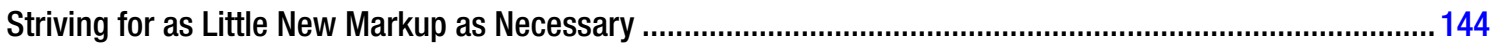

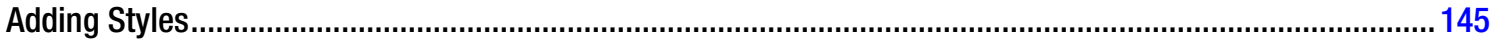

What About the Media Queries? .......................................................................................................... 147

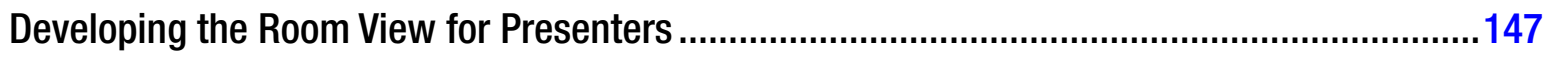

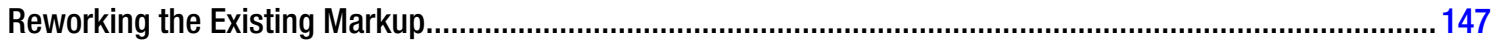

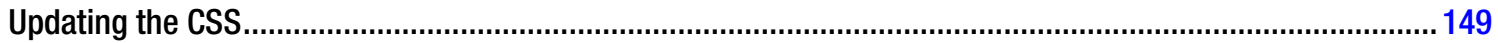

Updating the Media Queries ....................................................................................................... 151

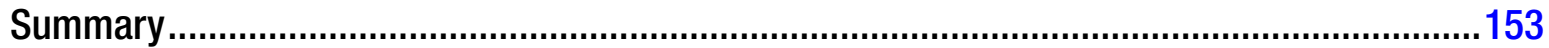

Chapter 8: Building the Back End: Part 1 .............................................................155

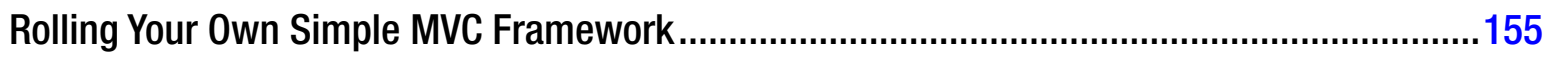

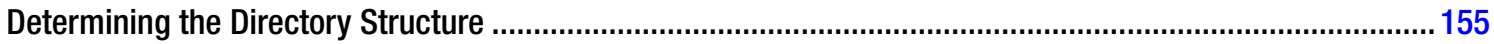

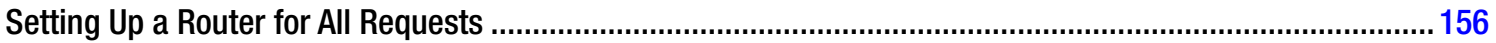

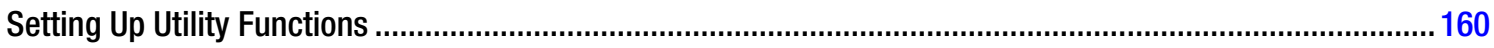

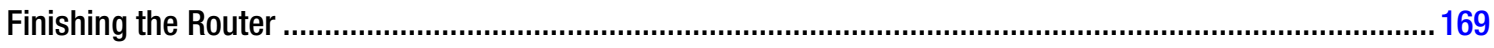

Setting Up the Core Classes ..............................................................................................................171

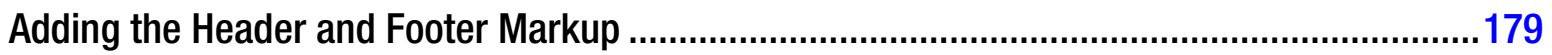

Setting Variables for the Header...................................................................................................... 181

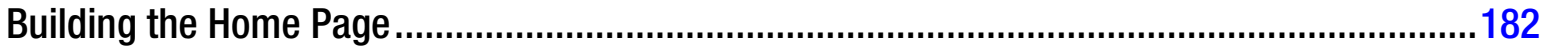

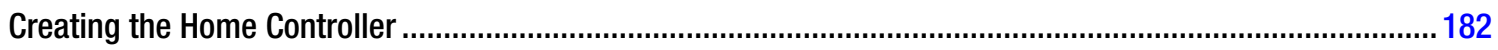

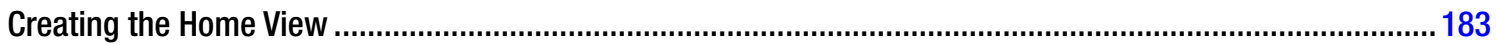

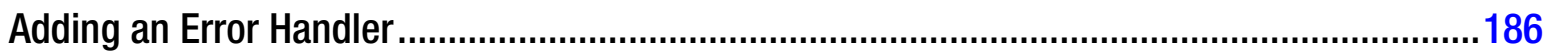

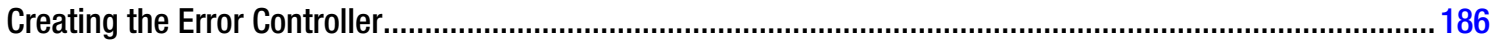

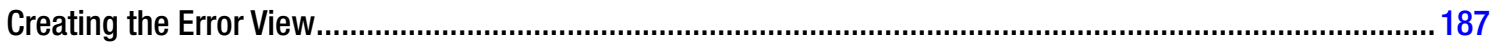

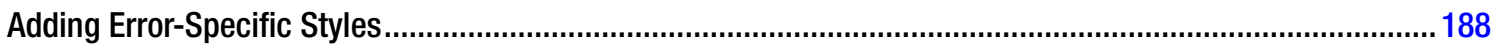

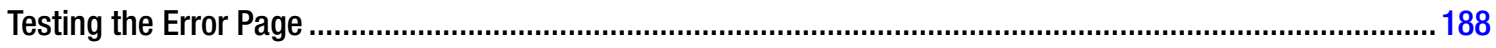

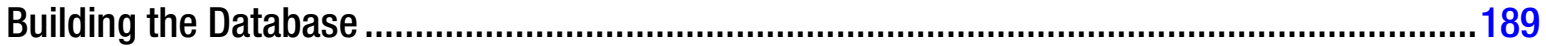

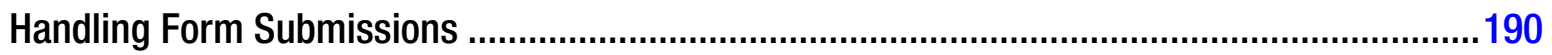

Planning the Form Submission Workflow............................................................................................. 190

Setting Up and Checking for Valid Actions...............................................................................................191 


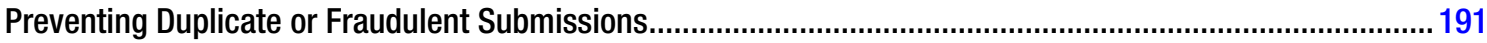

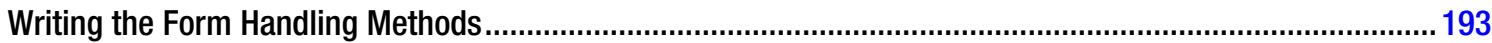

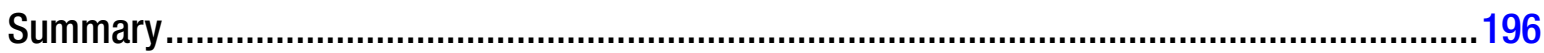

Chapter 9: Building the Back-End: Part 2 ................................................................197

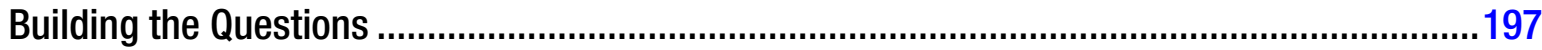

Building the Question Controller....................................................................................................... 197

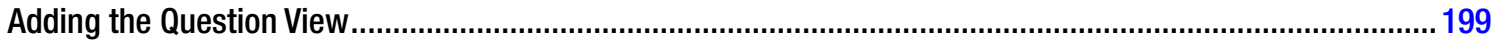

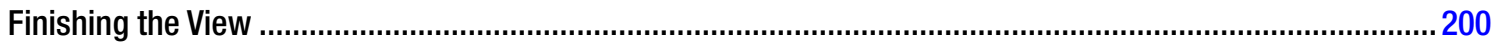

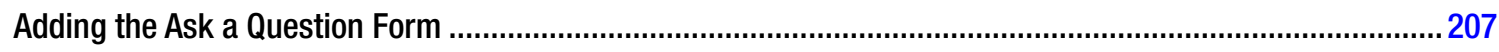

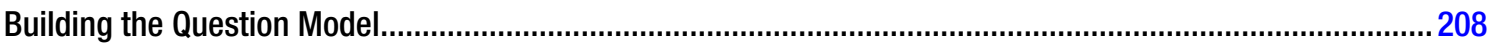

Adding Form Handlers and Data Access Methods to the Controller ........................................................213

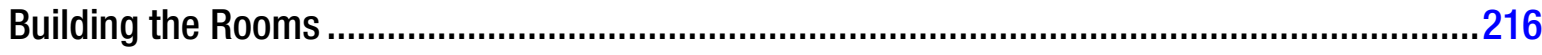

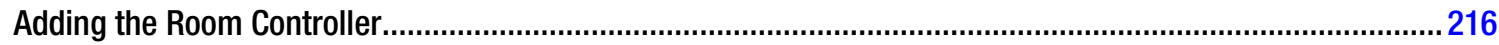

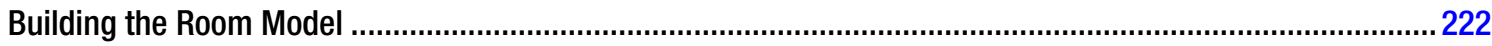

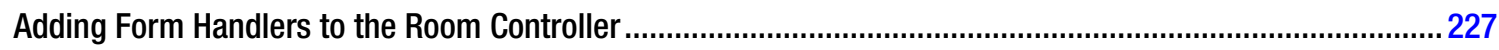

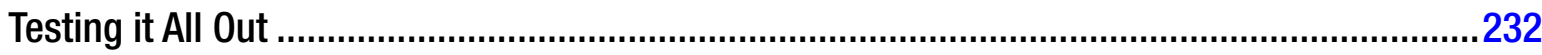

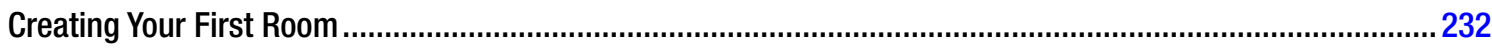

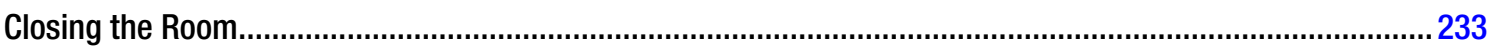

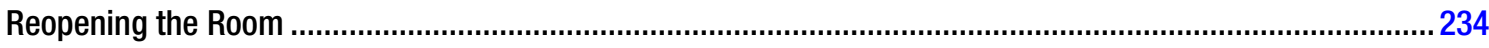

Joining a Room

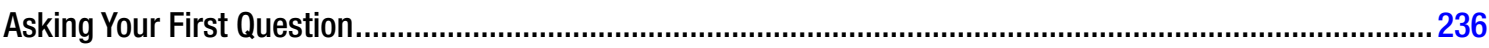

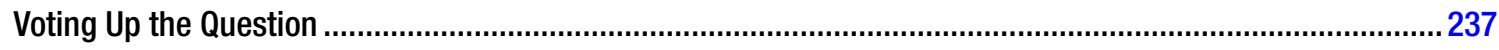

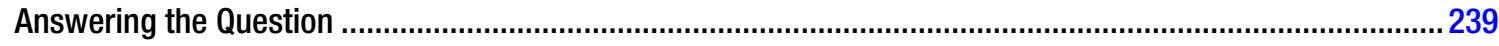

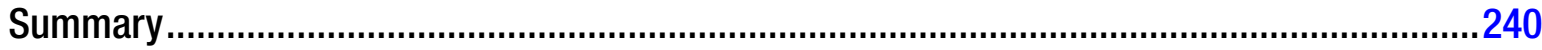

Chapter 10: Implementing Realtime Events and jQuery Effects ..................................241

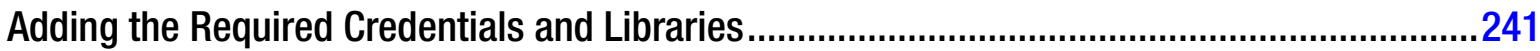

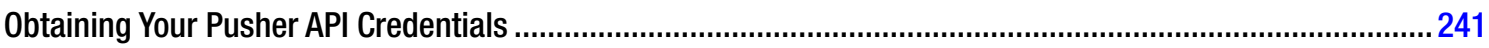

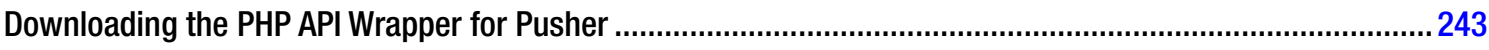

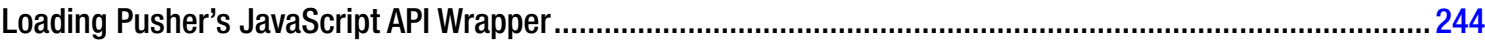

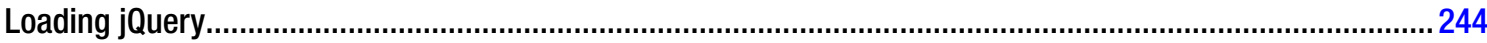




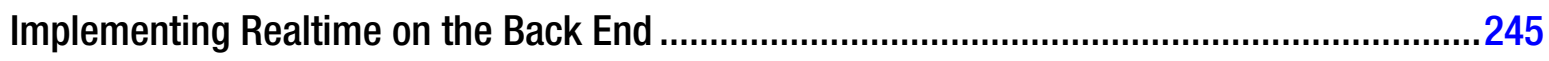

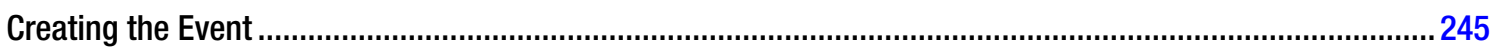

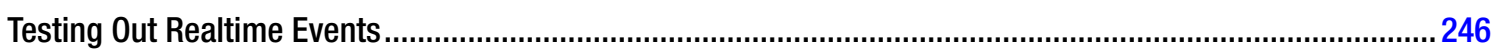

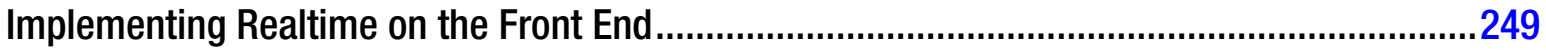

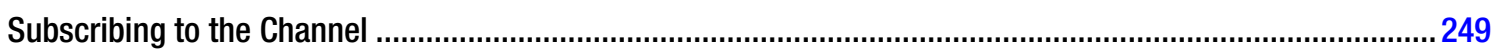

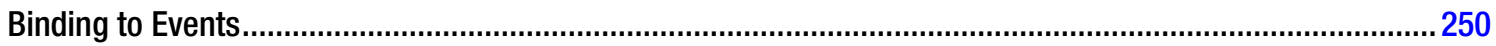

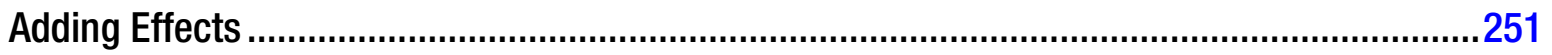

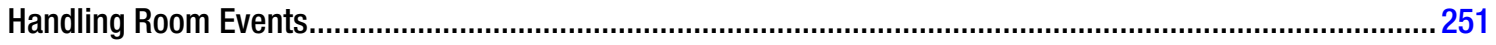

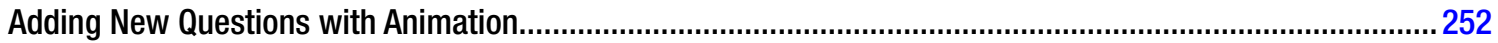

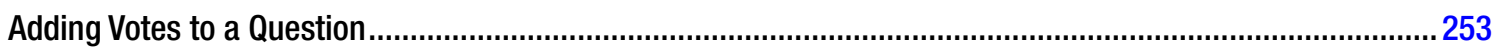

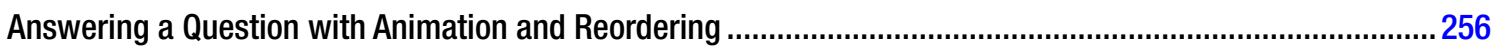

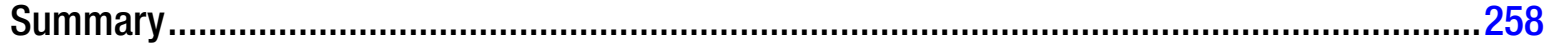

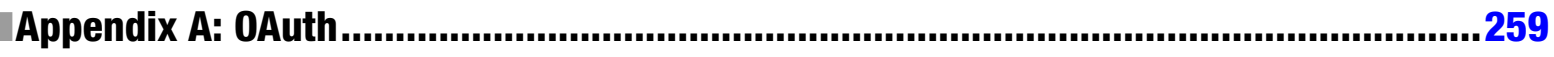

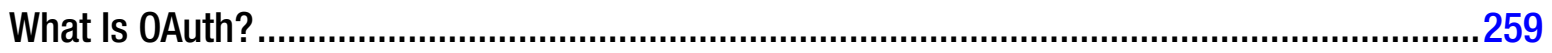

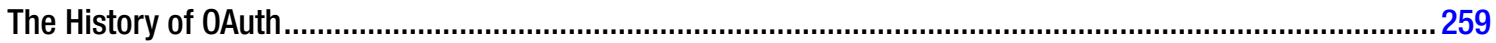

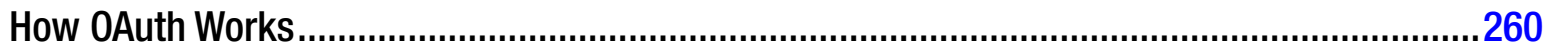

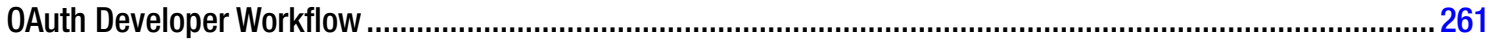

Why OAuth Is Better Than Building a Login System ...........................................................264

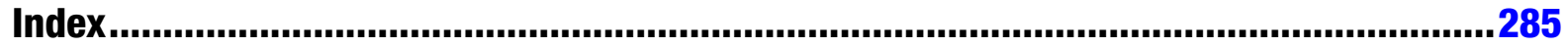




\section{About the Authors}

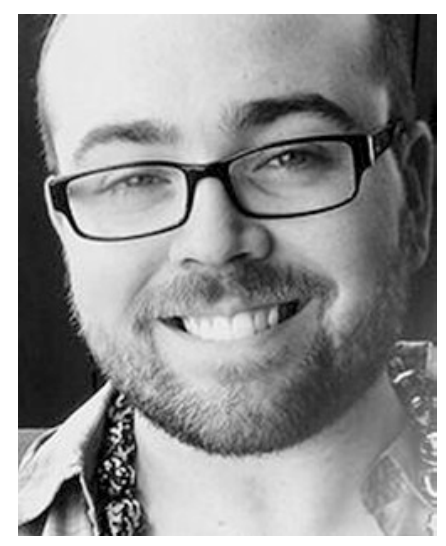

Jason Lengstorf is a 20-something from Portland, Oregon who has spent the last decade or so learning how to make a living without wearing pants. Along the way, he started Copter Labs, wrote a few books, drew a few pictures, and spoke to a few like-minded geeks. He's a music nerd, a foodie, a shameless coffee snob, and a big fan of wandering the globe.

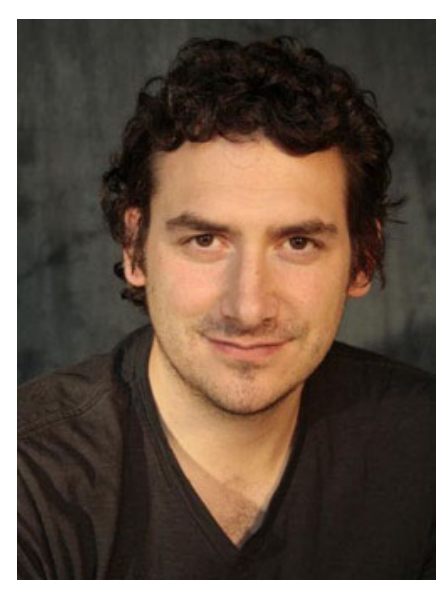

Phil Leggetter is a Developer Evangelist at Pusher and has been developing and using real-time web technologies for more than 10 years. His focus is to help people use these technologies to build the next generation of interactive and engaging real-time web applications. He does this through writing articles; creating sample open-source applications; running workshops; and speaking at universities, companies, and conferences. 


\section{Acknowledgments}

This book owes its very life to Phil Leggetter, who was able to keep things moving along. He added tons of background information about the technologies and did a great job of adding clear explanations and examples. Without his help, we'd probably still be stuck on the first half of the book.

To Ben and Ana, your patience was hugely appreciated.

To Alison, thank you for not leaving me when I stayed up all night writing.

To Nate, thanks for providing friendly competition and reminding me that there's always a next step once I've accomplished a goal.

To my dad, thanks for always being an inspiration. To my mom, thanks for putting up with me and Dad for all these years.

To Drew, thanks for coming on board at Copter Labs so I could clear time to work on this project. To Alex, Anne, Jason, Kevin, Rob, Roger, and Wes, thanks for being part of the Copter team.

To Richelle, Troy, Taunja, Chris, and all the other friends who help prevent me from becoming an unwashed hermit, thanks for continuing to invite me to do things. 


\section{Introduction}

A couple of years back, I went to a conference called "Keeping It Realtime." It was a collection of presenters who were deep in the trenches of the realtime world, solving problems that most of the rest of the world had never even heard about.

The power of this technology was staggering, and the number of places that it was already being used was pretty surprising. I wanted to know more, start using it right then. How could I start using this wonderful, magical new idea in my own applications?

I sat down in the audience for one of the hands-on sessions and was immediately lost. A small, shy dude with a beard was at the podium with his laptop, mumbling into the microphone and coding in Vim at incredible speeds. By the time I was able to figure out that he was initializing socket.io, he'd already gotten halfway through the meat of the app.

My spirits sank, and I started to wonder whether this kind of awesome technology was reserved only for that elite shadow group of secret ninja developers. If I can't keep up with a guy who is teaching this stuff, how am I ever supposed to build anything on my own?

If you've ever asked a really smart developer how to do something, you might know the feeling: when someone hits a certain level of smart, they can sometimes forget how to talk to the rest of us who haven't used that tech before. This puts us in a situation in which we can either dig through tons of complex code and specifications and rough documentation, or we can just give up.

This book is intended to help demystify realtime coding and make it accessible to any developer with medium PHP and JavaScript chops. If you want to use this stuff in real projects right now, and don't need to know how to build a Flash polyfill or maintain Node.js, this book is right up your alley.

We believe that while theory is fun and necessary, the real exciting part of development is in putting it to use and seeing it come to life. To that end, the technologies used in this book are simple to set up and don't require you to learn a new programming language or framework; the book is based on the same web technologies used in some of the most popular apps, websites, and content management systems out there today.

Realtime should belong to the caffeinated masses, so grab your coffee (or tea) and let's get started. You'll be up and running with realtime before it gets cold. 